\title{
Including Thermal Vibrations and Bonding in HAADF-STEM Image Simulation
}

\author{
Michael Odlyzko and K. Andre Mkhoyan
}

Chemical Engineering and Materials Science, University of Minnesota, Minneapolis, MN 55455

Conventional implementations of multislice TEM image simulation [1,2] model the electrostatic potential of a solid as that of a collection of unbonded independent atoms; this approximation is known as the "independent atom model" (IAM). It is acknowledged that IAM-based calculations introduce errors in the low-order atomic scattering factor [3], and accordingly several studies have shown that valence charge redistribution due to bonding significantly affects bright-field image contrast [4,5]. In a previous study, the authors showed that charge redistribution due to bonding alters the high-angle annular dark field (HAADF) scanning TEM (STEM) image contrast in light-element polar crystals by altering probe channeling [6]. However, that study neglected to consider the effect of thermal diffuse scattering, which quantitatively affects HAADF-STEM image contrast [7].

To understand the effect of thermal vibrations on bonding-inclusive HAADF-STEM image simulation, we have employed multislice simulations of STEM imaging of the light-element crystals LiF, BeO, $\mathrm{NaF}, \mathrm{MgO}$, and $\mathrm{AlN}$, all of which had exhibited strong bonding-model sensitivity without thermal vibrations. The TEMSIM multislice package [8] was used to simulate imaging in an aberration-corrected STEM (accelerating voltages 100 and $200 \mathrm{kV}$, probe convergence semi-angle 25 mrad, HAADF detector semi-angles 60-200 mrad). Multislice inputs were generated by parameterizing projected atomic potentials calculated via the Quantum Espresso package [9]. All materials were modeled at thicknesses up to $100 \mathrm{~nm}$ by IAM, then compared to samples modeled as bonded atoms by density functional theory in the "bonded crystal model" (BCM) and as independent full-valence-shell ions in the "fully ionized model" (FIM). Images and channeling profiles were simulated along zone axes with each column containing only one type of atom, using thermal vibration amplitudes determined from the experimental diffraction literature.

As demonstrated by the example of wurtzite AlN aligned to the $<\overline{2} 110>$ zone axis, the on-column channeling intensity (Figure 1) and quantitative comparisons of HAADF contrast (Figure 2) all indicate the sensitivity of HAADF-STEM image formation to bonding, both with and without thermal vibrations. Results show that thermal vibrations dampen but do not remove the effects of charge transfer due to bonding, an effect robust with respect to changing probe size and electron energy [10].

\section{References}

[1] M.A. O’Keefe et al., Nature 274 (1978), p. 322.

[2] E.J. Kirkland et al., Ultramicroscopy 23 (1987), p. 77.

[3] J.M. Zuo et al., J. Phys. Cond. Matter 9 (1997), p. 7541.

[4] B. Deng and L.D. Marks, Acta Cryst. A 62 (2006), p. 208.

[5] J.C. Meyer et al., Nature Materials 10 (2011), p. 209.

[6] M.L. Odlyzko and K.A. Mkhoyan, Proc. Microsc. Microanal. (2013), p. 602.

[7] Z.L. Wang and J.M. Cowley, Ultramicroscopy 31 (1989), p. 437.

[8] E.J. Kirkland, Advanced Computing in Electron Microscopy, (Springer, New York, 2010).

[9] P. Giannozzi et al., J. Phys. Cond. Matter 29 (2009), p. 395502. 
[10] This research was supported by NSF DMR-1006706. Simulations were performed using Minnesota Supercomputing Institute resources. Drs. M. Cococcioni and B. Himmetoglu are thanked for density functional theory calculations of bonding charge density.
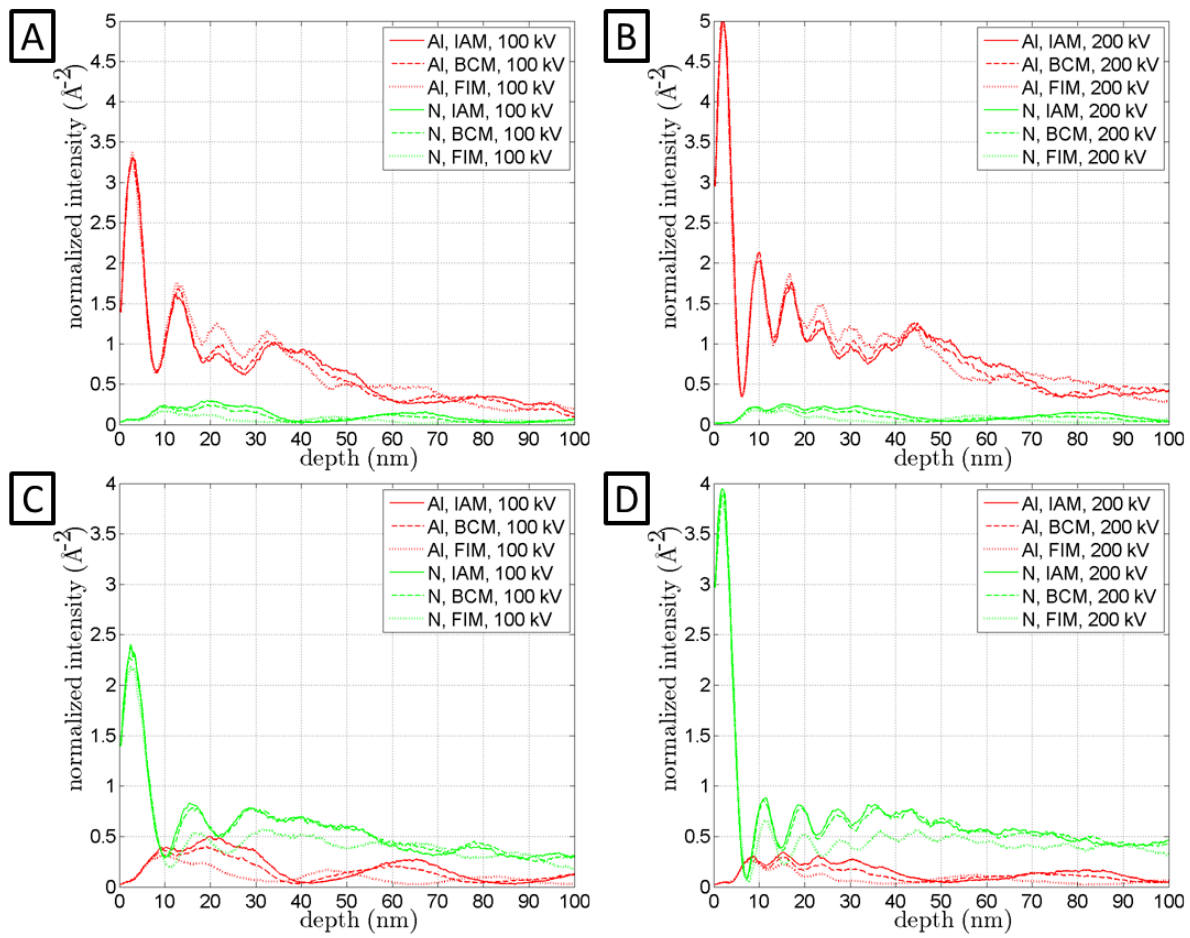

Figure 1. Channeling profiles in $<2110>$-oriented $\mathrm{AlN}$ at $300 \mathrm{~K}$ : (A) $100 \mathrm{kV}$ and (B) $200 \mathrm{kV}$ probes centered on an Al column, as well (C) $100 \mathrm{kV}$ and (D) $200 \mathrm{kV}$ probes centered on an $\mathrm{N}$ column; in all cases, the cumulative intensity on the three nearest neighboring columns is plotted together with the center column intensity. Introducing charge transfer between atoms raises intensity and increases the frequency of intensity oscillations on the net-positively-charged Al column, while decreasing the intensity and decreasing the oscillation frequency for the net-negatively-charged $\mathrm{N}$ column. Increasing charge transfer also weakens dechanneling from one column to another.
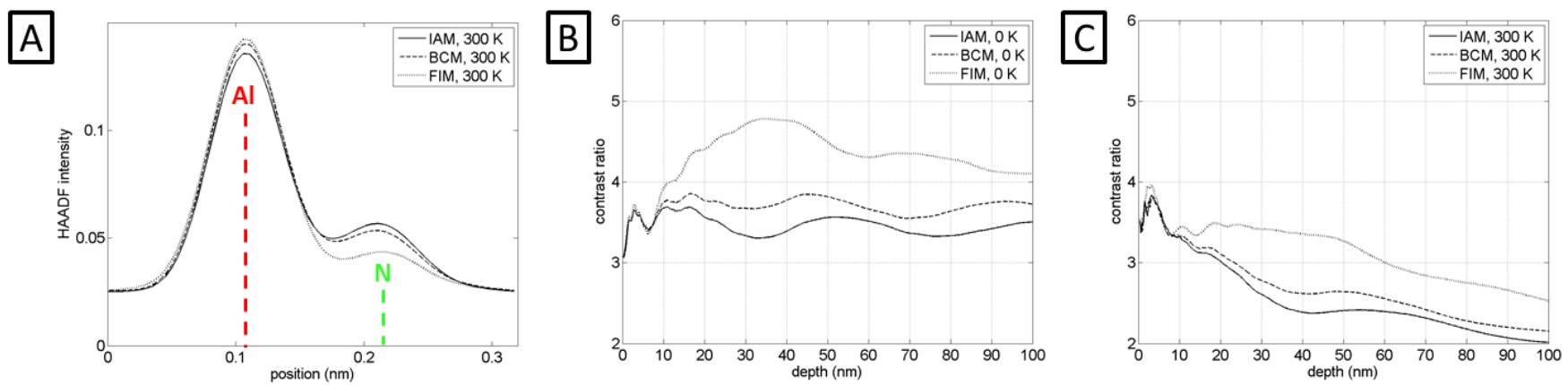

Figure 2. (A) Example linescan comparing HAADF imaging of $50 \mathrm{~nm}$ thick $<\overline{2} 110>$-oriented AlN by a $200 \mathrm{kV}$ probe for each bonding model at $300 \mathrm{~K}$. To capture the depth-dependence of image contrast, the contrast ratio (the ratio of intensity on the Al column to that on the $\mathrm{N}$ column, $\mathrm{I}_{\mathrm{Al}} / \mathrm{I}_{\mathrm{N}}$ ) is plotted as a function of depth for HAADF simulations of a $200 \mathrm{kV}$ probe imaging $<\overline{2} 110>$-oriented AlN (B) at 0 $\mathrm{K}$ and $(\mathrm{C})$ at $300 \mathrm{~K}$; for both BCM and FIM cases, both with and without phonons, beyond a depth of about $8 \mathrm{~nm}$ the image contrast deviates from that of the IAM model. 